\title{
A survey of 3.3 micron PAH emission in planetary nebulae using FLITECAM
}

\author{
Erin C. Smith ${ }^{1}$ and Ian S. McLean ${ }^{2}$ \\ University of California, Los Angeles, \\ Division of Astronomy and Astrophysics, 430 Portola Plaza, Los Angeles CA 90095 \\ email: ${ }^{1}$ erincds@astro.ucla.edu, ${ }^{2}$ mclean@astro.ucla.edu
}

\begin{abstract}
We have performed a study of 3.3 micron PAH emission in planetary nebulae using ground-based observations with FLITECAM, one of a suite of instruments designed for airborne astronomy aboard SOFIA, NASA's Stratospheric Observatory for Infrared Astronomy. The survey was performed on the Shane 3 meter telescope at Lick Observatory as part of the ground-based commissioning of the FLITECAM grism spectroscopy mode. Spectral resolution of $\mathrm{R} \sim 1700$ was obtained with direct-ruled KRS-5 grisms. Targets included AGB stars and sources showing PAH emission in KAO, ISO or IRAS observations. Additionally, several oxygen-rich nebulae were observed in order to test methodology. Twenty objects were surveyed, of which 11 showed PAH emission. In objects exhibiting PAH emission, the relationship between the nebular $\mathrm{C} / \mathrm{O}$ ratio and $\mathrm{PAH}$ equivalent width was found, showing a detectable $\mathrm{PAH}$ emission cutoff at a nebular $\mathrm{C} / \mathrm{O}$ ratio of $0.65 \pm 0.28$. Selected objects with detected PAH emission were further investigated to trace PAH emission spectral variation within individual nebulae.
\end{abstract}

Keywords. Instrumentation: spectrographs, planetary nebulae: general, (ISM:) dust, extinction

We have carried out a preliminary survey of $3.3 \mu \mathrm{m}$ PAH emission using FLITECAM on the Lick Observatory $3-\mathrm{m}$ telescope. FLITECAM is a 1-5 $\mu \mathrm{m}$ camera developed by us at the UCLA Infrared Laboratory (P.I.: McLean) for NASA's SOFIA (Stratospheric Observatory for Infrared Astronomy) project (Mainzer \& McLean 2003, McLean et al. 2006). SOFIA is a modified Boeing 747-SP airplane with a 2.5-meter f/19.6 bent-Cassegrain telescope operating at altitudes up to $45,000 \mathrm{ft}$ and therefore above $99 \%$ of the atmosphere's water vapor content.

This study of $3.3 \mu \mathrm{m}$ emission from PN was carried out as part of the commissioning and performance verification of the FLITECAM grism spectroscopy mode. This mode utilizes a suite of three KRS-5 grisms in conjunction with fixed slits of either 1 arcseconds or 2 arcseconds width and 60 arcseconds total length to achieve medium resolution ( $\mathrm{R} \sim 1700$ and 900) spectroscopy (Smith \& McLean 2006). An airborne survey similar to this will be carried out using the same instrument on board the Stratospheric Observatory for Infrared Astronomy (SOFIA).

Observations were carried out over the course of five observing runs at Lick observatory. Targets were selected from ISO, KAO and IRAS observations of planetary nebulae, proto-planetary nebulae and post-AGB stars (Jourdain de Muizon et al. 1990, Rinehart et al. 2002). Out of 20 objects observed, 11 showed detectable $3.3 \mu \mathrm{m}$ PAH emission. Telluric correction was achieved with the ATRAN atmosphere modeling package (Lord 1992). Each PAH detection was fitted with a gaussian to determine FWHM and central wavelength. We found the $3.3 \mu \mathrm{m}$ feature to have an average FWHM of $0.042 \mu \mathrm{m}$ $\pm 0.002 \mu \mathrm{m}$. and central wavelength of $3.288 \mu \mathrm{m} \pm 0.002 \mu \mathrm{m}$. These are consistent with values found by Tokunaga et al. (1991), Roche et al. (1996), and van Diedenhoven 
et al. (2004). All PAH emission was classified as type $\mathrm{A}_{3.3}$ according to the system proposed by Peeters et al. (2002) and van Diedenhoven et al. (2004).

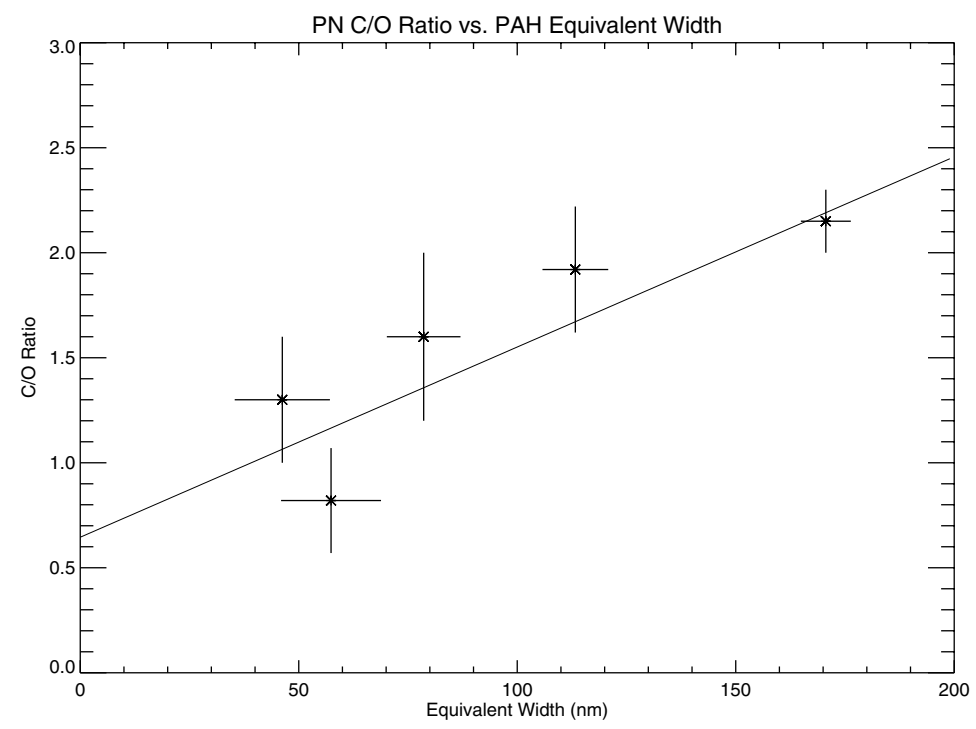

Figure 1. C/O Ratio versus PAH equivalent width.

The equivalent width of each $\mathrm{PAH}$ detection was also determined in order to investigate the correlation between the nebular $\mathrm{C} / \mathrm{O}$ ratio and the $\mathrm{PAH}$ emission strength. Figure 1 shows the derived $3.3 \mu \mathrm{m}$ emission equivalent width plotted against the nebular $\mathrm{C} / \mathrm{O}$ ratio obtained from the literature (Kholtygin 1998, Liu et al. 2004) and found a correlation between $\mathrm{C} / \mathrm{O}$ ratio and $\mathrm{PAH}$ emission strength. We calculate a threshold $\mathrm{C} / \mathrm{O}$ ratio of $0.65 \pm 0.28$, which is consistent with that reported by Roche et al. (1996) and Cohen \& Barlow (2005).

\section{References}

Cohen, M. \& Barlow, M. J. 2005, MNRAS, 362, 1199

Jourdain de Muizon, M., Cox, P., \& Lequeux, J. 1990, $A \& A S, 83,337$

Kholtygin, A. F. 1998, A\&A, 329, 691

Liu, Y., Liu, X.-W., Barlow, M. J., \& Luo, S.-G. 2004, MNRAS, 353, 1251

Lord, S. D. 1992, NASA TM103957

Mainzer, A. K. \& McLean, I. S. 2003, ApJ, 597, 555

McLean, I. S., Smith, E. C., Aliado, T., Brims, G., Kress, E., Magnone, K., Milburn, J., Oldag, A., Silvers, T., \& Skulason, G. 2006, in: I. S. McLean \& M. Iye (eds.), Proceedings of the SPIE, Volume 6269

Peeters, E., Hony, S., Van Kerckhoven, C., Tielens, A. G. G. M., Allamandola, L. J., Hudgins, D. M., \& Bauschlicher, C. W. 2002, A\&A, 390, 1089

Rinehart, S. A., Houck, J. R., Smith, J. D., \& Wilson, J. C. 2002, MNRAS, 336, 66

Roche, P. F., Lucas, P. W., Hoare, M. G., Aitken, D. K., \& Smith, C. H. 1996, MNRAS, 280, 924

Smith, E. C. \& McLean, I. S. 2006, in: I. S. McLean \& M. Iye (eds.), Proceedings of the SPIE, Volume 6269, p. 50

Tokunaga, A. T., Sellgren, K., Smith, R. G., Nagata, T., Sakata, A., \& Nakada, Y. 1991, ApJ, 380,452

van Diedenhoven, B., Peeters, E., Van Kerckhoven, C., Hony, S., Hudgins, D. M., Allamandola, L. J., \& Tielens, A. G. G. M. 2004, ApJ, 611, 928 